\title{
Grammognatha euphratica euphratica (Dejean, 1822) nell'isola di S. Antioco (Sardegna), nuova vistosa specie che si aggiunge alla fauna d'Italia
}

\author{
(Coleoptera: Cicindelidae $)^{1}$
}

\begin{abstract}
Riassunto: Una nuova, vistosa specie di Coleotteri, il Cicindelide Megacefalino Grammognatha euphratica euphratica (Dejean, 1822), rinvenuta nell'Isola di S. Antioco in Sardegna (Sulcis) da uno degli autori, si è recentemente e inaspettatamente aggiunta alla fauna d'Italia ed è segnalata per la prima volta nella presente nota.

Abstract: Grammognatha euphratica euphratica (Dejean, 1822) from S. Antioco island (Sardinia) a new species for italian fauna (Coleoptera: Cicindelidae).

A new, notable species of beetles, i.e. the megacephaline tiger beetle Grammognatha euphratica euphratica (Dejean, 1822), sampled in the S. Antioco island (Sardinia, Sulcis) by one of the authors, has been added, recently and unexpectedly, to the Italian fauna. It is reported for the first time in this contribution.
\end{abstract}

Key words: Coleoptera, Cicindelidae, Megacephalinae, Grammognatha euphratica, Italian fauna.

\section{INTRODUZIONE}

Il Cicindelide Megacefalino Grammognatha euphratica (Dejean, 1822) è un coleottero vistoso e di cospicue dimensioni (Fig. 1). La specie in senso lato è ben conosciuta di alcuni paesi del Mediterraneo: in Nord Africa dal Morocco alla Tunisia (Cassola, 1973a; Korell \& Cassola, 1987), Spagna (Jeanne, 1967; Jeanne \& Zaballos, 1986; Serrano, 2003; Zaballos \& Jeanne, 1994), Grecia (Cassola, 1973b; Cassola 1981, Creta), Turchia (Cassola, 1999; Franzen, 2002), Egitto e Medio Oriente fino all'India (Franzen, 2002), Cipro (Jeanne, 1986; Franzen \& Gigli, 2003), Francia (Jeannel, 1941; Forel \& Leplat, 1992; Coulon et al., 2011). Inoltre, è conosciuta di alcuni paesi africani, soprattutto dell'Africa occidentale (Cassola, 2004, 2007; Cassola \& Jaskula, 2006).

Non erano note fino a oggi segnalazioni in territorio politicamente italiano.

\section{MATERIALI E METODI}

Il 4 giugno 2013 uno degli autori della presente nota (P. Bonneau), mentre si trovava a campionare nel retroduna leggermente paludoso della spiaggia Cani- soni, nell'Isola di S. Antioco in Sardegna (Sulcis), scorgeva un individuo di Grammognatha euphratica euphratica (Dejean, 1822) che usciva dal suo foro scavato nel terreno e iniziava a correre in tutte le direzioni.

La sorprendente notizia è stata subito comunicata al primo Autore dal secondo Autore. Quasi contemporaneamente, la notizia era comunque pervenuta al primo Autore anche per altra via. Va ricordato che il primo Autore ha vissuto in Sardegna, a Cagliari, dal 1965 al 1970, e che in tale periodo ha avuto occasione di visitare personalmente $\mathrm{S}$. Antioco numerose volte, senza peraltro mai reperire tale specie, neanche sotto forma di semplici resti.

\section{DiSCUSSIONE E CONCLUSIONI}

Grammognatha euphratica è specie di non facile reperimento, a causa dei suoi costumi strettamente crepuscolari o francamente notturni, a meno che non ci si trovi già sul posto all'ora opportuna. Si comprende pertanto come, in un primo tentativo di censimento e valutazione della fauna italiana (Cassola, 1964), e di quella sarda in particolare (Cassola, 1972), la specie non sia risultata presente.

\footnotetext{
${ }^{1}$ Studi sui Cicindelidi. CLXXXVII.

*Fabio Cassola, Via F. Tomassucci 20, 00144 Roma, Italia. E-mail: fabiocassola@alice.it

${ }^{* *}$ Philippe Richoux, 35 Allée des Prunus, 69140 Rillieux-la-Pape, France. E-mail: richoux.philippe@wanadoo.fr

${ }^{* * *}$ Patrick Bonneau, 3 Lotissement Les Vignes, Ancien chemin d'Aix, 13720 La Bouilladisse, France. E-mail: alstor.pb@sfr.fr
} 


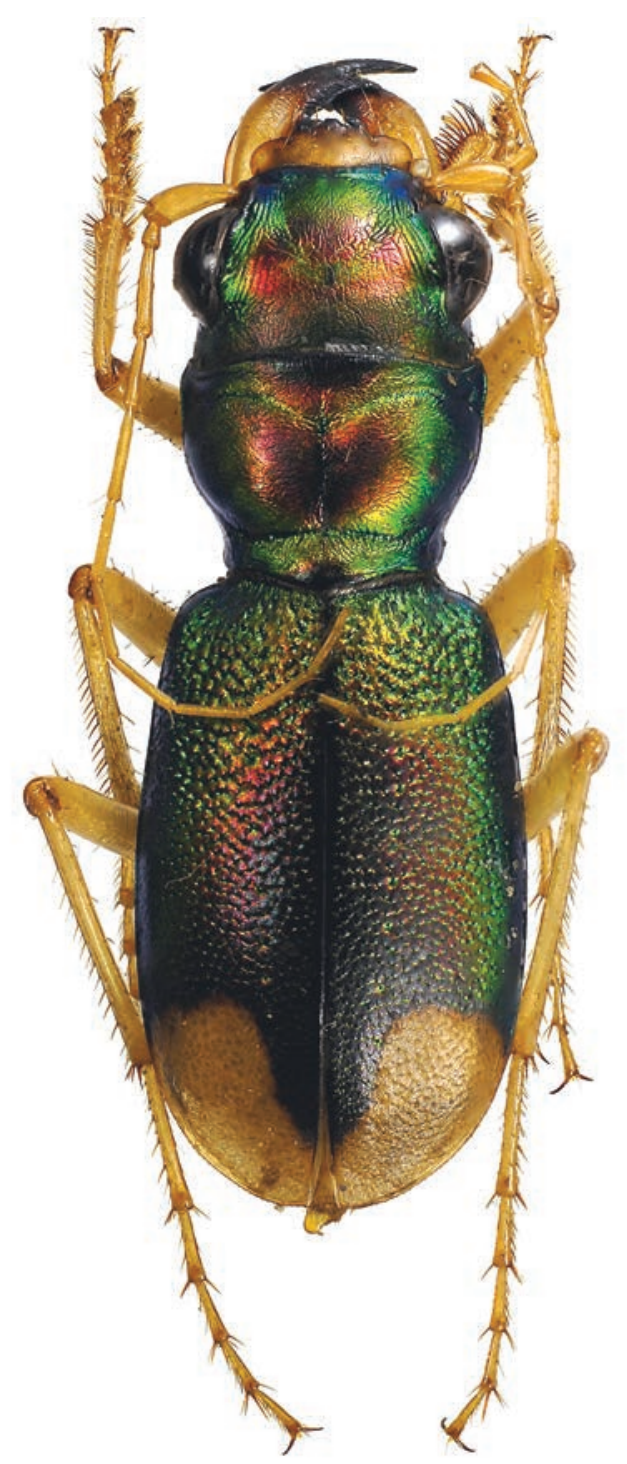

Fig. 1. Grammognatha euphratica euphratica (Dejean, 1822). Photo P. Bonneau.
Un metodo relativamente efficace per catturare di giorno questa specie è comunque quello di individuare dapprima l'entrata di un foro (generalmente, almeno all'inizio, orizzontale) sul bordo del sedimento sabbioso-argilloso indurito, generalmente indicato da minuscole palline di sedimento davanti all'ingresso (risultato dello scavo del foro, che diviene poi rapidamente verticale). E' poi sufficiente scavare la galleria dietro l'apertura per spingere l'individuo ad uscire allo scoperto, dove risulta facile catturarlo. Questo metodo si è dimostrato ad esempio molto efficace all'estuario della Moulouya (Oujda, Marocco), durante le raccolte effettuate da Philippe Richoux et Guy Chavanon (Chavanon \& Chavanon, 1992).

Il recente ritrovamento restituisce all'Italia, dopo la Turchia ed ex-aequo con la Spagna, unitamente alle Lophyra sarda [sardea (Dejean, 1831)] e siciliana [circumflexa (Dejean, 1831)], nelle quali il primo autore tende sempre più a riconoscere per vari motivi specie distinte dalla congenere flexuosa (Fabricius, 1787) (Cassola, 2011), il primato nel numero di specie di Cicindelidi (19) facenti parte della propria fauna nell'area mediterranea. Il dato è comprensibile e congruente con la posizione geografica della penisola italica, vero ponte sotteso al centro del Mediterraneo, capace di intercettare e ospitare componenti faunistiche provenienti e insediate da nord come da sud, e da est come da ovest. 
BIBLIOGRAFIA

Cassola F., 1964 - Note su alcuni Cicindelidi italiani (Coleoptera Cicindelidae). Bollettino dell'Associazione Romana di Entomologia, 19(2): 18-20.

Cassola F., 1970 - The Cicindelidae of Italy. Cicindela, 2(4): 1-20.

Cassola F., 1972 - Studi sui Cicindelidi. V. Il popolamento della Sardegna (Coleoptera Cicindelidae). Studi sassaresi, (3), Annali della Facoltà di Agraria dell’Università di Sassari, 20: 3-42, tavv. I-IV.

Cassola F., 1973a - Contribution à la connaissance des Cicindélides du Maroc (Coleoptera: Cicindelidae). Bulletin de la Société de Sciences Naturelles et Physiques du Maroc, Rabat, 53: 253-268.

CAssola F., 1973b - Études sur les Cicindelides. X. Matériaux pour un catalogue des Cicindelidae de Grèce (Coleopters). Biologia gallo-hellenica, Athénes, 5: 25-141.

Cassola F., 1981 - Studi sui Cicindelidi. XXVII. Una notevole aggiunta alla faunadi Creta: Megacephala euphratica (Dejean) (Coleoptera: Cicindelidae). Fragmenta Entomologica, Roma, 16(1): 25-30.

CAssola F., 1999 - Le Cicindele come indicatori ambientali (Coleoptera: Cicindelidae) (Studi sui Cicindelidi. C. Atti dell'Accademia Nazionale di Entomologia (Atti del Simposio coordinato da A. Vigna Taglianti \& A. Casale, Firenze 27 novembre 1998), Firenze, 1999, 46: 337-352. [Ripubblicato anche in: Accademia Nazionale di Entomologia, Filogenesi e sistematica dei Carabidi, Firenze 2004, 363 pp. 337-352].

Cassola F., 2004 - Studies of Tiger Beetles. CLII. Grammognata euphratica (Dejean, 1831) new for Senegal (Coleoptera: Cicindelidae). Mitteilungen des Internationalen Entomologischen Vereins, Frankfurt a.M., 29(3/4): 71-76.

Cassola F., 2007 - Studies of Tiger Beetles. CLXI. New data from Benin (Coleoptera: Cicindelidae). Rivista Piemontese di Storia Naturale, 28: 157-164.

CASsola F., 2011 - Studies of Tiger Beetles (XXXII congresso S.I.B., Catania 7-10 ottobre 2008). On the Sicilian Lophyra populations. Biogeographia, 30: 635-640.

Cassola F., Jaskula R., 2006 - New records of Tiger Beetles from Burkina Faso (Coleoptera: Cicindelidae). Mitteilungen des Internationalen Entomologischen Vereins, Frankfurt a.M., 5(1/2): 5-58.

Chavanon G., Chavanon L., 1992 - Etude sur la Basse Moulouya (Maroc oriental). I. Les Coléoptères carabiques de l'embouchure (Caraboidea). Bulletin mensuel de la Société linnéenne de Lyon, 61: 39-45.

Coulon J., Pupier R., Queinnec E., Ollivier E., Richoux P., 2011 - Coléoptères Carabiques. Compléments aux deux volumes de René Jeannel, et mise à jour, corrections et repertoire. Faune de France 94-95. Fédération française des sociétés d'histoire naturelle, pp. 684.

Forel J., Leplat J., 1992 - Faune des Carabiques de France -1. Collection Systèmatique 5, Magellanes, Andresy: 1-97.

FranZEN M., 2002 - Distribution of the tiger beetle Megacephala (Grammognatha) euphratica in Egypt, the Middle East and central Asia (Coleoptera: Cicindelidae). Zoology in the Middle East, 22: 87-93.

FranZEN M., GigLi M., 2003 - Rediscovery of the tiger beetle Megacephala euphratica on Cyprus (Coleoptera: Cicindelidae). Zoology in the Middle East, 28: 127-128.

Jeanne C., 1967 - Carabiques de la Péninsule Ibèrique (4e note). Mèmoires Société linnéenne de Bordeaux, 104(3): 4-34.

JEANNE C., 1986 - Contribution à l'histoire naturelle de l'ile de Chypre. Les Coleoptères Carabiques. Biocosme mésogéen, 3(1): $1-33$.

Jeanne C., Zaballos J.P., 1986 - Catalogue des Coléoptères Carabiques de la Péninsule Ibérique. Bullettin de la Société Linnéenne de Bordeaux suppl: pp. 200

JEANNEL R., 1941 - Faune de France 39 Coléoptères carabiques. t1. Lechevalier, Paris, pp. 571.

Korell A., Cassola F., 1987 - Über die Sandlaufkäfer Tunisiens. Mitteilungen Münchner Entomologische Gesellschaft, 77: 85-121.

Serrano J., 2003 - Catalogo de los Carabidae (Coleoptera) de la Peninsula Iberica. Monografias SEA, 9: pp. 130.

Zaballos J.P., Jeanne C., 1994 - Nuevo Catalogo de los Carabidos de la Penìnsula Ibèrica. Monografias SEA, 9: 1-159. 